\title{
Pacific
}

Journal of

Mathematics

UNIT INDICES OF SOME IMAGINARY COMPOSITE QUADRATIC FIELDS. II

MiKinito HiRABAYASHI 


\title{
UNIT INDICES OF SOME IMAGINARY COMPOSITE QUADRATIC FIELDS II
}

\author{
Mikihito HiRABAYASHI
}

Let $K$ be an imaginary abelian number field of type $(2,2,2,2)$ containing the 8-th cyclotomic field $\mathbf{Q}(\sqrt{-1}, \sqrt{2})$. Using the fundamental units of real quadratic subfields of $K$, we give a necessary and sufficient condition for the unit index $Q_{K}$ of $K$ to be equal to 2 .

\section{Introduction and Results.}

Let $K$ be an imaginary abelian number field and $K_{0}$ the maximal real subfield of $K$. Let $E$ and $E_{0}$ be the groups of units of $K$ and $K_{0}$, respectively, and let $W$ be the group of roots of unity in $K$. Let $Q_{K}$ be the unit index of $K$, i.e.,

$$
Q_{K}=\left[E: W E_{0}\right]
$$

In the previous paper [4] we gave a necessary and sufficient condition for $Q_{K}$ to be equal to 2 when $K$ is an imaginary abelian number field (whose Galois group is) of type $(2,2,2,2)$ not containing the 8-th cyclotomic field $\mathbf{Q}(\sqrt{-1}, \sqrt{2})$. In this paper we give such a condition when $K$ contains $\mathbf{Q}(\sqrt{-1}, \sqrt{2})$.

In this paper we use the following notation, unless otherwise specified.

$\mathbf{N}, \mathbf{Z}, \mathbf{Q}$ : the sets of natural numbers, rational integers and rational numbers, respectively,

$\overline{\overline{2}}($ resp. $\overline{2}$ in $k)$ : the equality up to a rational quadratic factor (resp. the equality up to a square of a number of a field $k$ ),

$d_{1}, d_{2}, \cdots, d_{7}$ : square-free positive integers such that $d_{4}=d_{2} d_{3}, d_{5}=d_{3} d_{1}$, $d_{6}=d_{1} d_{2}, d_{7}=d_{1} d_{2} d_{3}$ and that $d_{3}=2$.

$K=\mathbf{Q}\left(\sqrt{-1}, \sqrt{d_{1}}, \sqrt{d_{2}}, \sqrt{d_{3}}\right)=\mathbf{Q}\left(\sqrt{-1}, \sqrt{2}, \sqrt{d_{1}}, \sqrt{d_{2}}\right)$ : an imaginary abelian number field of type $(2,2,2,2)$,

$K_{0}=\mathbf{Q}\left(\sqrt{d_{1}}, \sqrt{d_{2}}, \sqrt{d_{3}}\right)$,

$E_{0}^{+}$: the group of totally positive units of $K_{0}$,

$$
\begin{array}{ll}
K_{1}=\mathbf{Q}\left(\sqrt{d_{2}}, \sqrt{d_{3}}\right), \quad K_{2}=\mathbf{Q}\left(\sqrt{d_{3}}, \sqrt{d_{1}}\right), \quad K_{3}=\mathbf{Q}\left(\sqrt{d_{1}}, \sqrt{d_{2}}\right), \\
K_{4}=\mathbf{Q}\left(\sqrt{d_{1}}, \sqrt{d_{2} d_{3}}\right), \quad K_{5}=\mathbf{Q}\left(\sqrt{d_{2}}, \sqrt{d_{3} d_{1}}\right), K_{6}=\mathbf{Q}\left(\sqrt{d_{3}}, \sqrt{d_{1} d_{2}}\right), \\
K_{7}=\mathbf{Q}\left(\sqrt{d_{2} d_{3}}, \sqrt{d_{3} d_{1}}\right) &
\end{array}
$$


$\sigma_{i}$ : a generator of $\operatorname{Gal}\left(K_{0} / K_{i}\right)$, i.e., $\left\langle\sigma_{i}\right\rangle=\operatorname{Gal}\left(K_{0} / K_{i}\right) \quad(i=1,2, \cdots, 7)$,

$\varepsilon_{i}$ : the fundamental unit of $k_{i}=\mathbf{Q}\left(\sqrt{d_{i}}\right), \varepsilon_{i}>1 \quad(i=1,2, \cdots, 7)$,

$N(x), S p(x)$ : the absolute norm and the absolute trace of an algebraic number $x$, respectively.

For a totally positive unit $\eta$ of $K_{0}$, let

$$
\begin{aligned}
& \xi=\xi(\eta)=\eta+\eta^{\sigma_{1}}+2 \sqrt{\eta \eta^{\sigma_{1}}} \\
& \theta=\theta(\eta)=\xi+\xi^{\sigma_{2}}+2 \sqrt{\xi \xi^{\sigma_{2}}}
\end{aligned}
$$

under the condition that

$$
\sqrt{\eta \eta^{\sigma_{1}}} \in K_{1} \text { and } \sqrt{\xi \xi^{\sigma_{2}}} \in k_{3} .
$$

Let $\nu$ be the number of $i$ for which $N\left(\varepsilon_{i}\right)=-1(i=1,2, \cdots, 7)$, i.e.,

$$
\nu=\#\left\{i \mid i=1,2, \cdots, 7 ; N\left(\varepsilon_{i}\right)=-1\right\} \text {. }
$$

Remark 1. Using Lemmas 3 and 6 we can show that the above condition (3) follows from the equations

$$
N_{K_{0} / K_{i}}(\eta)=1 \quad \text { in } K_{i} \quad(i=1,2,6) .
$$

Our result is

Theorem. (1) If $\nu \geq 4$, then $Q_{K}=1$.

(2) Suppose that $\nu=3$ and that

$$
N\left(\varepsilon_{s}\right)=N\left(\varepsilon_{t}\right)=N\left(\varepsilon_{3}\right)=-1
$$

for $s, t \in\{1,2, \cdots, 7\}(s \neq t)$ different from 3 . If $d_{s} d_{t}=\frac{d_{3}}{3}$ does not hold, then $Q_{K}=1$.

(3) Suppose that $\nu \leq 2$ or that $\nu=3$ and $d_{s} d_{t}=d_{3}$ holds for above $s, t$. Then $Q_{K}=2$ if and only if there exists a unit $\eta$ in $E_{0}^{+}$such that

$$
\eta=\prod_{i=1}^{7} \varepsilon_{i}^{a_{i}} \cdot \sqrt{\prod_{N\left(\varepsilon_{j}\right)=+1} \varepsilon_{j}^{b_{j}}} \quad\left(a_{i}, b_{j}=0 \text { or } 1\right)
$$

satisfying the following conditions (i), (ii) :

(i)

$$
N_{K_{0} / K_{\alpha}}(\eta)=1 \quad \text { in } K_{\alpha} \quad(\alpha=1,2,6)
$$

$$
N_{K_{0} / K_{\beta}}(\eta)=1 \quad \text { in } K_{0} \text {, but not in } K_{\beta} \quad(\beta=3,4,5,7) \text {. }
$$


(ii)

$$
\theta=\theta(\eta)=(2+\sqrt{2}) d_{1}^{e_{1}} d_{2}^{e_{2}} \text { in } k_{3}=\mathbf{Q}(\sqrt{2})
$$

for some $e_{i} \in\{0,1\}$.

Moreover, in the representation (4) of $\eta$, the number of $j$ 's for which $b_{j}=1$ is greater than one.

Remark 2. When $\nu=3$ and $d_{s} d_{t}=d_{3}$ holds for $s, t$ in Theorem, we have examples of $Q_{K}=1$ and $Q_{K}=2$ :

If $d_{1}=5, d_{2}=21$, then $Q_{K}=1$, which is checked by Proposition 1 .

If $d_{1}=7, d_{2}=41$, then $Q_{K}=2$. Because,

$$
\eta=\sqrt{\varepsilon_{1}} \sqrt{\varepsilon_{5}}=\frac{1}{2}(3 \sqrt{2}+\sqrt{14}) \cdot(2 \sqrt{2}+\sqrt{7})
$$

satisfies the condition (3) of Theorem. In fact,

$$
\theta=\theta(\eta)=(2+\sqrt{2}) 7 \text { in } k_{3} .
$$

Remark 3. In the Theorem, when

$$
\prod_{N\left(\varepsilon_{j}\right)=+1} \varepsilon_{j}^{b_{j}}=\varepsilon_{j_{1}} \varepsilon_{j_{2}}
$$

it holds that $d_{j_{1}} d_{j_{2}}=d_{3}=2$, as seen in Lemma 5 (2).

The assertions (1) and (2) of the Theorem are easily obtained in $\S 3$ from

Proposition 1. Let $L$ be the composite of a 2-power-th cyclotomic field $\mathbf{Q}(\zeta)\left(\zeta=\exp \left(2 \pi i / 2^{m}\right), m \geq 2\right)$ and $n$ independent real quadratic fields $\mathbf{Q}\left(\sqrt{D_{i}}\right)$ where $D_{\imath}$ are square-free positive integers $(i=1,2, \cdots, n)$, that is,

$$
L=\mathbf{Q}\left(\zeta, \sqrt{D_{1}}, \sqrt{D_{2}}, \cdots, \sqrt{D_{n}}\right) .
$$

If $D_{1} \equiv D_{2} \equiv \cdots \equiv D_{n} \equiv 1(\bmod 4)$, then $Q_{L}=1$.

\section{Characterization of $\eta \in \bar{E}_{0}$.}

Our argument depends on

Lemma 1 (cf. [3, Satz 15]). $Q_{K}=2$ if and only if there exists a unit $\eta \in E_{0}^{+}$such that $K_{0}(\sqrt{\eta})=K_{0}(\sqrt{2+\sqrt{2}})$.

Therefore, in order to determine the alternative $Q_{K}=1$ or 2 , we investigate such $\eta \in E_{0}^{+}$. We replace the definition of $\bar{E}_{0}$ in [4] by

$$
\bar{E}_{0}=\left\{\eta \in E_{0}^{+} \mid K_{0}(\sqrt{\eta})=K_{0}(\sqrt{2+\sqrt{2}})\right\} .
$$


Here we note that if $\eta \in \bar{E}_{0}, \eta$ is totally positive.

Lemma 2 (cf. [4, Lemma 1]). For $\eta \in \bar{E}_{0}$, we have

$$
\eta^{2}=\varepsilon_{1}^{x_{1}} \varepsilon_{2}^{x_{2}} \cdots \varepsilon_{7}^{x_{7}}
$$

for some $x_{i} \in \mathbf{Z}$.

Proof. For $\eta \in \bar{E}_{0}$, we can put

$$
\eta^{4}=\varepsilon_{1}^{x_{1}} \varepsilon_{2}^{x_{2}} \cdots \varepsilon_{7}^{x_{7}} \quad\left(x_{i} \in \mathbf{Z}\right) .
$$

In fact, for a $(2,2)$-extension $K / k$ with Galois group $\operatorname{Gal}(K / k)=\langle\sigma, \tau\rangle$ we have

$$
\alpha^{2}=\frac{\alpha^{1+\sigma} \alpha^{1+\tau}}{\left(\alpha^{\sigma}\right)^{1+\sigma \tau}}
$$

for any $\alpha \in K, \alpha \neq 0$. By this formula we see that $E_{0}^{4} \subseteq E_{0}^{*}$, where $E_{0}^{*}$ is the subgroup of $E_{0}$ generated by $\pm \varepsilon_{i} \quad(i=1,2, \cdots, 7)$.

We show that every $x_{i}$ is even.

Since $K_{0}(\sqrt{\eta})=K_{0}(\sqrt{2+\sqrt{2}})$, we have $\eta=(2+\sqrt{2}) \alpha_{0}^{2}$ for some $\alpha_{0} \in K_{0}$. Then

$$
(2+\sqrt{2})^{4} \alpha_{0}^{8}=\varepsilon_{1}^{x_{1}} \varepsilon_{2}^{x_{2}} \cdots \varepsilon_{7}^{x_{7}} .
$$

Taking the norms $N_{K_{0} / k_{3}}$ and $N_{K_{0} / k_{i}}(i \neq 3)$ of this equation (5) and then the positive fourth root, we have

$$
(2+\sqrt{2})^{4} N_{K_{0} / k_{3}}\left(\alpha_{0}\right)^{2}=\varepsilon_{3}^{x_{3}} \text { and } 2^{2} N_{K_{0} / k_{i}}\left(\alpha_{0}\right)^{2}=\varepsilon_{i}^{x_{2}}
$$

respectively. Here we recall that $\varepsilon_{3}$ and $\varepsilon_{i}$ are positive. These equations show that $\varepsilon_{i}^{x_{2}}$ is square in $k_{i}$ and hence $x_{i} \equiv 0(\bmod 2)$ for every $i$.

Lemma 3 ([2, Satz 1]). Let $K_{1}$ be a field with $\operatorname{char}\left(K_{1}\right) \neq 2$ and $K_{0}$ a quadratic extension over $K_{1}$. Let $\eta$ be an element of $K_{0}$ which is not a square in $K_{0}$.

(1) $K_{0}(\sqrt{\eta}) / K_{1}$ is Galois $\Longleftrightarrow N_{K_{0} / K_{1}}(\eta)=1$ in $K_{0}$.

(2) $K_{0}(\sqrt{\eta}) / K_{1}$ is an extension of type $(2,2) \Longleftrightarrow N_{K_{0} / K_{1}}(\eta)=1$ in $K_{1}$.

(3) $K_{0}(\sqrt{\eta}) / K_{1}$ is cyclic $\Longleftrightarrow N_{K_{0} / K_{1}}(\eta)=1$ in $K_{0}$, but not in $K_{1}$.

Lemma 4 (cf. [4, Lemma 3]). Let $\eta \in \bar{E}_{0}$ and put

$$
\eta^{2}=\varepsilon_{1}^{x_{1}} \varepsilon_{2}^{x_{2}} \cdots \varepsilon_{7}^{x_{7}} \quad\left(x_{i} \in \mathbf{Z}\right) .
$$


(1) If there exists an even $x_{i}$, then $N\left(\varepsilon_{j}\right)=+1$ for each odd $x_{j}$.

(2) If $x_{1} \equiv x_{2} \equiv \cdots \equiv x_{7} \equiv 1(\bmod 2)$, then $N\left(\varepsilon_{1}\right)=N\left(\varepsilon_{2}\right)=\cdots=$ $N\left(\varepsilon_{7}\right)$.

We can prove this Lemma 4 as in the same way in [4, Lemma 3].

Lemma 5. Let $\eta \in \bar{E}_{0}$ and put

$$
\eta^{2}=\varepsilon_{1}^{x_{1}} \varepsilon_{2}^{x_{2}} \cdots \varepsilon_{7}^{x_{7}} \quad\left(x_{i} \in \mathbf{Z}\right) .
$$

(1) There exist at least two odd integers among the $x_{i}$ 's.

(2) If $x_{i}, x_{j}(i \neq j)$ are odd and the others $x_{k}$ are even, then $d_{i} \neq 2, d_{j} \neq 2$ and $d_{i} d_{j}=2$.

Proof of Lemma 5. (1) First we suppose that all $x_{\imath}$ are even. Then $\eta$ is a product of some of $\varepsilon_{i}$ 's. Noting that $\eta$ is contained in $\left(E_{0}^{*}\right)^{+}=E_{0}^{*} \cap E_{0}^{+}$, we see by $[4$, Proposition 1$]$ that $\eta$ is, up to a square, a product of some of following totally positive units :

$$
\begin{aligned}
& \left.\varepsilon_{i} \quad \text { (when } N\left(\varepsilon_{i}\right)=+1\right) \\
& \begin{array}{ll}
\eta_{i j}:=\varepsilon_{i} \varepsilon_{j} \varepsilon_{k} & \text { (when } \left.d_{\imath} d_{j}=d_{k} \text { and } N\left(\varepsilon_{i}\right)=N\left(\varepsilon_{j}\right)=N\left(\varepsilon_{k}\right)=-1\right) \\
\eta_{i j k}:=\varepsilon_{i} \varepsilon_{j} \varepsilon_{k} \varepsilon_{l} & \text { (when } d_{i} d_{j} d_{k}=d_{l} \text { and } N\left(\varepsilon_{i}\right)=N\left(\varepsilon_{j}\right)=N\left(\varepsilon_{k}\right)=N\left(\varepsilon_{l}\right) \\
& =-1) .
\end{array}
\end{aligned}
$$

For a unit $\varepsilon_{i}$ with $N\left(\varepsilon_{i}\right)=+1$ we have

$$
\eta S p(\xi)=\xi^{2}
$$

where $\eta=\varepsilon_{i}$ and $\xi=\varepsilon_{i}+1$. For $\eta=\eta_{\imath j}$ or $\eta_{i j k}$ we also have by [5, Proof of Zusatz 1] or by [4, Lemma 6] that

$$
\eta S p(\xi)=\xi^{2}
$$

where

$$
\xi=\varepsilon_{i} \varepsilon_{j} \varepsilon_{k}-\varepsilon_{i}-\varepsilon_{j}-\varepsilon_{k}
$$

or

$$
\xi=\varepsilon_{i} \varepsilon_{j} \varepsilon_{k} \varepsilon_{l}+1-\left(\varepsilon_{i} \varepsilon_{j}+\varepsilon_{j} \varepsilon_{k}+\varepsilon_{k} \varepsilon_{i}+\varepsilon_{i} \varepsilon_{l}+\varepsilon_{j} \varepsilon_{l}+\varepsilon_{k} \varepsilon_{l}\right),
$$

respectively. Therefore, $K_{0}\left(\sqrt{\varepsilon_{i}}\right), K_{0}\left(\sqrt{\eta_{i j}}\right)$ and $K_{0}\left(\sqrt{\eta_{i j k}}\right)$ are 2-elementary extensions over $\mathbf{Q}$ and so is $K_{0}(\sqrt{\eta})$, which contradicts $\eta \in \bar{E}_{0}$.

Next we suppose that $x_{i}$ is odd and the other $x_{k}$ are even. Choose $K_{j}$ for which $\sqrt{d_{i}} \notin K_{j}$. Taking the norm $N_{K_{0} / K_{j}}$ of the equation (6), we have

$$
N_{K_{0} / K_{j}}(\eta)^{2}=N\left(\varepsilon_{i}\right)^{x_{\imath}} \varepsilon_{u}^{2 x_{u}} \varepsilon_{v}^{2 x_{v}} \varepsilon_{w}^{2 x_{w}}
$$


where $K_{j}=\mathbf{Q}\left(\sqrt{d_{u}}, \sqrt{d_{v}}\right)$ and $d_{w}=d_{u} d_{v}$. Hence, $N\left(\varepsilon_{i}\right)=+1$ and so $i \neq 3$. (Then, as for above $j$, we can take $j=3,4,5$ or 7.) Moreover, since $x_{u}, x_{v}$ and $x_{w}$ are even, we have

$$
N_{K_{0} / K_{j}}(\eta)=\varepsilon_{u}^{x_{u}} \varepsilon_{v}^{x_{v}} \varepsilon_{w}^{x_{w}}=1 \text { in } K_{j} .
$$

Therefore it follows from Lemma 3 that $K_{0}(\sqrt{\eta}) / K_{j}$ is of type $(2,2)$. However, the extension $K_{0}(\sqrt{\eta}) / K_{j}=K_{0}(\sqrt{2+\sqrt{2}}) / K_{j}$ is itself a cyclic extension of degree 4 . Thus we get a contradiction.

(2) Choose $k \in\{1,2, \cdots, 7\}$ for which $\sqrt{d_{i}} \in K_{k}$ and $\sqrt{d_{j}} \notin K_{k}$. Taking the norm $N_{K_{0} / K_{k}}$ of the equation (6), we have

$$
N_{K_{0} / K_{k}}(\eta)^{2}=\varepsilon_{i}^{2 x_{i}} N\left(\varepsilon_{j}\right)^{x_{\jmath}} \eta_{k}^{2}
$$

where $\eta_{k}$ is a unit of $K_{k}$. Hence $N\left(\varepsilon_{j}\right)=+1$ and so $d_{j} \neq d_{3}=2$.

By exchanging $i$ and $j$, we also have $N\left(\varepsilon_{i}\right)=+1$ and $d_{i} \neq d_{3}$.

Finally we show that $d_{i} d_{j}=2$. Assume that this is false. Then, $K_{l}:=$ $\mathbf{Q}\left(\sqrt{d_{i} d_{3}}, \sqrt{d_{j} d_{3}}\right)$ contains neither $\sqrt{d_{i}}$ nor $\sqrt{d_{j}}$. Taking the norm $N_{K_{0} / K_{l}}$ of (6) and then the positive square root, we obtain

$$
N_{K_{0} / K_{l}}(\eta)=\varepsilon_{\alpha}^{x_{\alpha}} \varepsilon_{\beta}^{x_{\beta}} \varepsilon_{\gamma}^{x_{\gamma}}{ }_{2}=1 \quad \text { in } K_{l}
$$

where $d_{\alpha}=d_{i} d_{3}, d_{\beta}=d_{j} d_{3}$ and $d_{\gamma}=d_{\alpha} d_{\beta}$, because, $x_{\alpha}, x_{\beta}$ and $x_{\gamma}$ are even. Therefore, it follows from Lemma $3(2)$ that $K_{0}(\sqrt{\eta}) / K_{l}$ is an extension of type $(2,2)$. However, by the definition of $K_{l}, K_{l}$ does not contain $\sqrt{d_{3}}$ and so $K_{l} \neq K_{1}, K_{2}$ or $K_{6}$. Hence $K_{0}(\sqrt{\eta}) / K_{l}$ is a cyclic extension of degree 4 , which is a contradiction.

\section{Proofs of Proposition 1 and Theorem.}

Proof of Proposition 1. Let $f(\chi)$ be the conductor of a Dirichlet character $\chi$. For any even character $\chi_{0}$ of $L$, we have $2 \nmid f\left(\chi_{0}\right)$ or $2^{3} \mid f\left(\chi_{0}\right)$ and $2^{m+1} \backslash f\left(\chi_{0}\right)$. Then, from [2, Satz 22] it follows that $Q_{L}=1$.

Remark 4. Proposition 1 is also proved in [1 (14.7) Corollary and the comment on p. 87 - 88].

Proof of (1),(2) of Theorem. By the assumption we have

$$
K=\mathbf{Q}\left(\sqrt{-1}, \sqrt{2}, \sqrt{d_{s}}, \sqrt{d_{t}}\right), \quad N\left(\varepsilon_{s}\right)=N\left(\varepsilon_{t}\right)=N\left(\varepsilon_{3}\right)=-1
$$

for suitable $d_{s}, d_{t} \neq d_{3}$. Then for every odd prime $p$ dividing $d_{s} d_{t}$, we have $p \equiv 1(\bmod 4)$. In fact, for example, by $N\left(\varepsilon_{s}\right)=-1$ we have $x^{2}-d_{s} y^{2}=-4$ 
for some $x, y \in \mathbf{Z}$. Then, for an odd prime $p$ dividing $d_{s}, x^{2} \equiv-4(\bmod p)$ and hence $(-1 / p)=(-1)^{\frac{p-1}{2}}=1$, where $(/)$ is the Legendre symbol. Thus we get $p \equiv 1(\bmod 4)$.

Therefore

$$
K=\mathrm{Q}\left(\sqrt{-1}, \sqrt{2}, \sqrt{D_{s}}, \sqrt{D_{t}}\right)
$$

for some $D_{s}, D_{t} \in \mathbf{N}, D_{s} \equiv D_{t} \equiv 1(\bmod 4)$. Thus Proposition 1 implies that $Q_{K}=1$.

In the following we prove the assertion (3) of Theorem, for which we need

Proposition 2. Let $K$ and $K_{0}$ be as in the notation in $\S 1$. Let $\eta$ be an element of $K_{0}$ which is not square in $K_{0}$.

(1) $K_{0}(\sqrt{\eta}) / \mathbf{Q}$ is a Galois extension if and only if

$$
N_{K_{0} / K_{i}}(\eta)=1 \text { in } K_{0} \quad(i=1,2, \cdots, 7) .
$$

(2) $K_{0}(\sqrt{\eta}) / \mathbf{Q}$ is an abelian extension of type $(2,2,2,2)$ if and only if

$$
N_{K_{0} / K_{i}}(\eta)=1 \text { in } K_{i} \quad(i=1,2, \cdots, 7) .
$$

(3) $K_{0}(\sqrt{\eta}) / \mathbf{Q}$ is an abelian extension of type $(2,2,4)$ and $K_{0}(\sqrt{\eta}) / k_{3}$ of type $(2,2,2)$ if and only if

$$
\left\{\begin{array}{c}
N_{K_{0} / K_{\alpha}}(\eta)=1 \text { in } K_{\alpha} \quad(\alpha=1,2,6), \\
N_{K_{0} / K_{\beta}}(\eta)=1 \quad \text { in } K_{0}, \text { but not in } K_{\beta}(\beta=3,4,5,7) .
\end{array}\right.
$$

Remark 5. This Proposition 2 remains valid if $K_{0}=\mathbf{Q}\left(\sqrt{2}, \sqrt{d_{1}}, \sqrt{d_{2}}\right)$ is replaced by $K_{0}=\mathbf{Q}\left(\sqrt{d_{1}}, \sqrt{d_{2}}, \sqrt{d_{3}}\right)$ with arbitrary $d_{3} \in \mathbf{N} \quad\left(d_{3}\right.$ : square-free, $d_{3} \geq 2$ ). Therefore, the condition (8) leads to the condition (5) of $[4]$.

For the proof of Proposition 2, we need the following two lemmas.

Lemma 6. Let $k$ be an algebraic number field. Let $K_{0} / k$ be an abelian extension of type $(2,2)$. Let $K_{1}, K_{2}$ and $K_{3}$ be the intermediate fields of $K_{0} / k$. Let $\eta$ be an element of $K_{0}$.

(1) $K_{0}(\sqrt{\eta}) / k$ is a Galois extension if and only if 


$$
N_{K_{0} / K_{i}}(\eta) \underset{2}{=1} \quad \text { in } K_{0} \quad(i=1,2,3) .
$$

(2) Suppose that $K_{0}(\sqrt{\eta}) / k$ is a Galois extension. Let

$$
\mu=\#\left\{i \mid i=1,2,3 ; N_{K_{0} / K_{i}}(\eta)=1 \text { in } K_{i}\right\}
$$

Then, $K_{0}(\sqrt{\eta}) / k$ is quaternion, abelian of type $(2,4)$, dihedral or abelian of type $(2,2,2)$ if and only if $\mu=0,1,2$ or 3 , respectively.

Lemma 7. Let $G$ be a group of order 16. Assume that there exists a normal subgroup $N$ of $G$ of order 2 with quotient group $G / N$ of type $(2,2,2)$. Then $G$ is isomorphic to one of the followings :

(a) a 2-elementary group

(b) an abelian group of type $(2,2,4)$

(c) a central product of an abelian subgroup $A$ and a dihedral or quaternion subgroup $B$ of order 8 such that $A B=G, A \cap B=N$. ( $A$ is the center of G.)

Lemma 6 is an immediate consequence of Lemma 3. Lemma 7 is a special case of $[6,(4.16)$ and Theorem 4.18].

Proof of Proposition 2. (1) Suppose that $K_{0}(\sqrt{\eta}) / \mathbf{Q}$ is a Galois extension. Then, for any quadratic subfield $k$ of $K_{0}, K_{0}(\sqrt{\eta}) / k$ is also a Galois extension. Hence, by Lemma 6 (1) we have

$$
N_{K_{0} / K_{i}}(\eta)=1 \text { in } K_{0}
$$

for every intermediate field $K_{i}$ of $K_{0} / k$.

Conversely, suppose that the condition (7) is satisfied. For an automorphism $\sigma$ of the algebraic closure $\overline{\mathbf{Q}}$ of $\mathbf{Q}$, the restriction $\sigma_{K_{0}}$ of $\sigma$ to $K_{0}$ belongs to the Galois group $\operatorname{Gal}\left(K_{0} / \mathbf{Q}\right)=\left\{\sigma_{0}=1, \sigma_{1}, \cdots, \sigma_{7}\right\}$. Then

$$
\sigma_{K_{0}}=\sigma_{i}
$$

for some $i$. By the assumption, we have

$$
\eta \eta^{\sigma_{i}}=\eta_{i}^{2}
$$

for some $\eta_{i} \in K_{0}$. Therefore,

$$
\sqrt{\eta}^{\sigma}= \pm \sqrt{\eta^{\sigma}}= \pm \frac{\eta_{i}}{\sqrt{\eta}}
$$

is contained in $K_{0}(\sqrt{\eta})$ and whence $K_{0}(\sqrt{\eta}) / \mathbf{Q}$ is a Galois extension. 
(2), (3) At first, we suppose that $K_{0}(\sqrt{\eta}) / \mathbf{Q}$ is a Galois extension with Galois group $G$. Let $N$ be the subgroup of $G$ corresponding to $K_{0}$.

Here we assume that $G$ is not abelian. Then, it follows from Lemma 7 that $G$ is a central product of an abelian subgroup $A$ and a non-abelian subgroup $B$ of degree 8 . Let $k$ be the subfield of $K_{0}(\sqrt{\eta})$ corresponding to $B$. Since $A \cap B=N$ and since $B$ is of order $8, k$ is a quadratic subfield of $K_{0}$, i.e., $k=k_{a}$ for some $a \in\{1,2, \cdots, 7\}$. Then, $K_{0}(\sqrt{\eta}) / k_{a}$ is a quaternion or dihedral extension. Let $K_{i}^{\prime}(i=1,2,3)$ be the intermediate fields of $K_{0} / k_{a}$ and let

$$
\mu=\#\left\{i \mid N_{K_{0} / K_{\imath}^{\prime}}(\eta) \underset{2}{=1} \text { in } K_{\imath}^{\prime}\right\} .
$$

Then, by Lemma 6 (2) we have $\mu=0$ or 2 .

Now, suppose that the condition (9) is satisfied. Then, $K_{0}(\sqrt{\eta}) / \mathrm{Q}$ is a Galois extension with Galois group $G$. If $G$ is not abelian, then, for above $\mu$ and $a$, we have by the condition (9) that $\mu=3$ or 1 according as $a=3$ or not, which is a contradiction. Therefore $G$ must be abelian.

Moreover, the equations

$$
N_{K_{0} / K_{\beta}}(\eta)=1 \quad \operatorname{not} \text { in } K_{\beta} \quad(\beta=3,4,5,7)
$$

imply that $K_{0}(\sqrt{\eta}) / K_{\beta}$ is cyclic. Hence it follows from Lemma 7 that $K_{0}(\sqrt{\eta}) / \mathbf{Q}$ is an abelian extension of type $(2,2,4)$. And the equations

$$
N_{K_{0} / K_{\alpha}}(\eta)=1 \quad \text { in } K_{\alpha} \quad(\alpha=1,2,6)
$$

imply that $K_{0}(\sqrt{\eta}) / k_{3}$ is an abelian extension of type $(2,2,2)$.

Next, suppose that the condition (8) is satisfied. In a similar way we see that $K_{0}(\sqrt{\eta}) / \mathrm{Q}$ is an abelian extension.

We show that $K_{0}(\sqrt{\eta}) / \mathbf{Q}$ is of type $(2,2,2,2)$. Assume that this is false, i.e., assume that $K_{0}(\sqrt{\eta}) / \mathbf{Q}$ is of type $(2,2,4)$. Let, as above,

$$
G=\operatorname{Gal}\left(K_{0}(\sqrt{\eta}) / \mathbf{Q}\right), N=\operatorname{Gal}\left(K_{0}(\sqrt{\eta}) / K_{0}\right)
$$

Then,

$$
G / N \cong \operatorname{Gal}\left(K_{0} / \mathbf{Q}\right)
$$

is of type $(2,2,2)$. By the assumption there exists an element $\sigma$ of $G$ of order 4. Since the order of the coset $\sigma N$ of $G / N$ is at most $2, \sigma^{2}$ is contained in $N$. Hence $N=\left\langle\sigma^{2}\right\rangle$, because $N$ has order 2. Let $K_{i}$ be the subfield of $K_{0}$ corresponding to $\langle\sigma\rangle$. Then $K_{0}(\sqrt{\eta}) / K_{\imath}$ is cyclic. Hence, by Lemma 3 (3), we have

$$
N_{K_{0} / K_{\imath}}(\eta)=1 \text { not in } K_{i}
$$


which is a contradiction to the condition (8).

Thus we have proved the sufficiencies of (2) and (3) of Proposition 2.

Conversely, their necessities are immediately deduced from Lemma 3 .

For the proof of (3) of Theorem, we also need

Lemma 8 ([4, Lemma 5]). Let $K_{1}$ be an algebraic number field and $K_{0}$ a quadratic extension of $K_{1}$. Let $K_{0}\left(\sqrt{\eta_{0}}\right) \quad\left(\eta_{0} \in K_{0}, \eta_{0} \notin K_{1}\right)$ be a biquadratic bicyclic extension of $K_{1}$ with $\operatorname{Gal}\left(K_{0}\left(\sqrt{\eta_{0}}\right) / K_{1}\right)=\langle\sigma, \tau\rangle$ and $\operatorname{Gal}\left(K_{0}\left(\sqrt{\eta_{0}}\right) / K_{0}\right)=\langle\tau\rangle$. Let $F$ be the intermediate field of $K_{0}\left(\sqrt{\eta_{0}}\right) / K_{1}$ fixed by $\sigma$. Then we have

$$
F=K_{1}\left(\sqrt{\eta_{0}}+{\sqrt{\eta_{0}}}^{\sigma}\right)
$$

Proof of (3) of Theorem. Suppose that $Q_{K}=2$. Then, by Lemma 1 there exists a unit $\eta$ in $E_{0}^{+}$such that

$$
K_{0}(\sqrt{\eta})=K_{0}(\sqrt{2+\sqrt{2}}) .
$$

By Lemma 2 we have

$$
\eta^{2}=\varepsilon_{1}^{x_{1}} \varepsilon_{2}^{x_{2}} \cdots \varepsilon_{7}^{x_{7}}
$$

for some $x_{i} \in \mathbf{Z} \quad(i=1,2, \cdots, 7)$. And we see by Lemma 5 (1) that there are at least two odd integers among $x_{i}$ 's.

If all $x_{i}$ are odd, then it follows from Lemma 4 (2) that

$$
N\left(\varepsilon_{1}\right)=N\left(\varepsilon_{2}\right)=N\left(\varepsilon_{3}\right)=\cdots=N\left(\varepsilon_{7}\right)=-1,
$$

and so $\nu=7$, which contradicts our assumption $\nu \leq 3$. Then there exists at least one even integer among $x_{i}$ 's. Hence Lemma 4 (1) implies that $N\left(\varepsilon_{i}\right)=+1$ for odd $x_{i}$. Therefore we may represent the $\eta$ in question as

$$
\eta=\prod_{i=1}^{7} \varepsilon_{i}^{a_{i}} \cdot \sqrt{\prod_{N\left(\varepsilon_{j}\right)=+1} \varepsilon_{j}^{b_{j}}} \quad\left(a_{i}, b_{j}=0 \text { or } 1\right)
$$

and Lemma 5 (1) shows that there are at least two $b_{j}=1$.

Since $K_{0}(\sqrt{\eta})=K_{0}(\sqrt{2+\sqrt{2}})$ is an extension of type $(2,2,4)$ over $\mathbf{Q}$ and of type $(2,2,2)$ over $k_{3}=\mathbf{Q}(\sqrt{2})$, Proposition 2 (3) implies the condition (3) (i) of Theorem. 
Moreover, it follows from Lemma 8 that $K_{1}(\sqrt{\xi})=K_{1}\left(\sqrt{\eta_{0}} \pm \sqrt{\eta_{0}}{ }^{\sigma}\right)$ is the intermediate field of $K_{0}(\sqrt{\eta}) / K_{1}$ fixed by $\sigma$ or $\tau \sigma$, where $\sigma$ is an automorphism of $\overline{\mathbf{Q}}$ over $\mathbf{Q}$ such that $\sigma_{K_{0}}=\sigma_{1},\left\langle\sigma_{1}\right\rangle=\operatorname{Gal}\left(K_{0} / K_{1}\right)$ and $\tau$ is a generator of $\operatorname{Gal}\left(K_{0}(\sqrt{\eta}) / K_{0}\right)$. Consequently we have $K_{1}(\sqrt{\xi}) \neq K_{0}$. Similary we can show that $k_{3}(\sqrt{\theta})$ is an intermediate field of $K_{1}(\sqrt{\xi}) / k_{3}$ and that $k_{3}(\sqrt{\theta}) \neq K_{1}$. Therefore

$$
k_{3}(\sqrt{\theta})=k_{3}\left(\sqrt{(2+\sqrt{2}) d_{1}^{e_{1}} d_{2}^{e_{2}}}\right)
$$

for some $e_{i} \in\{0,1\}$. Thus we obtain the condition (3) (ii) of Theorem.

Conversely, suppose that there exists a unit $\eta \in E_{0}^{+}$satisfying the conditions (3) (i), (ii) of Theorem. Then, it follows from Proposition 2 (3) that $K_{0}(\sqrt{\eta})$ is of type $(2,2,4)$ over $\mathbf{Q}$ and of type $(2,2,2)$ over $k_{3}=\mathbf{Q}(\sqrt{2})$. By Lemma 8, we see that $K_{1}(\sqrt{\xi})$ is an intermediate field of $K_{0}(\sqrt{\eta}) / K_{1}$ and $K_{1}(\sqrt{\xi}) \neq K_{0}$. Then we have

$$
K_{0}(\sqrt{\eta})=K_{0}(\sqrt{\xi})
$$

In the same way we get

$$
K_{1}(\sqrt{\xi})=K_{1}(\sqrt{\theta})
$$

Therefore,

$$
K_{0}(\sqrt{\eta})=K_{0}(\sqrt{\xi})=K_{0}(\sqrt{\theta}) .
$$

By the condition (3) (ii) of Theorem we have

$$
K_{0}(\sqrt{\theta})=K_{0}(\sqrt{2+\sqrt{2}}) .
$$

Thus we obtain

$$
K_{0}(\sqrt{\eta})=K_{0}(\sqrt{2+\sqrt{2}})
$$

from which Lemma 1 implies $Q_{K}=2$, as desired.

Acknowledgement. The author would like to thank Professor Ken-ichi Yoshino for his many helpful comments. The author would also like to express his hearty thanks to the referee for his or her various suggestions.

\section{References}

[1] P. E. Conner and J. Hurrelbrink, Class Number Parity, World Sientific, Singapore, 1988. 
[2] F. Halter-Koch, Arithmetische Theorie der Normalkörper von 2-Potenzgrad mit Diedergruppe, J. Number Theory, 3 (1971), 412-443.

[3] H. Hasse, Über die Klassenzahl abelscher Zahlkörper, Academie Verlag, Berlin, 1952 (reproduction: Springer Verlag, (1985)).

[4] M. Hirabayashi, Unit Indices of Some Imaginary Composite Quadratic Fields, Pacific J. Math., 164 (1994), 87-104.

[5] T. Kubota, Über den Bizyklischen Biquadratischen Zahlkörper, Nagoya Math. J., 10 (1956), 65-85.

[6] M. Suzuki, Group Theory II, Springer-Verlag, New York-Berlin-Heidelberg-Tokyo, 1986.

Received September 17, 1993. This research was partially supported by Grant-in-Aid for Science Research No.05640072, the Ministry of Education, Science and Culture, Japan.

Kanazawa Institute of Technology

7-1, OGIGAOKA, NONOICHI-MACHI

ISHIKAWA 921 , JAPAN 


\title{
PACIFIC JOURNAL OF MATHEMATICS
}

Founded by

\author{
$\begin{array}{ll}\text { E. F. Beckenbach (1906-1982) F. Wolf (1904-1989) } & \text { F }\end{array}$
}

EDITORS

Sun-Yung A. Chang (Managing Editor) Robert Finn

University of California

Los Angeles, CA 90095-1555

pacific@math.ucla.edu

F. Michael Christ

University of California

Los Angeles, CA 90095-1555

christ@math.ucla.edu

Nicholas Ercolani

University of Arizona

Tucson, AZ 85721

ercolani@math.arizona.edu
Stanford University

Stanford, CA 94305

finn@gauss.stanford.edu

Steven Kerckhoff

Stanford University

Stanford, CA 94305

spk@gauss.stanford.edu

Martin Scharlemann

University of California

Santa Barbara, CA 93106

mgscharl@math.ucsb.edu
Gang Tian

Massachusettes Institute of Technology

Cambridge, MA 02139

tian@math.mit.edu

\section{S. Varadarajan}

University of California

Los Angeles, CA 90095-1555

vsv@math.ucla.edu

Dan Voiculescu

University of California

Berkeley, CA 94720

dvv@math.berkeley.edu

\section{SUPPORTING INSTITUTIONS}

\author{
CALIFORNIA INSTITUTE OF TECHNOLOGY \\ NEW MEXICO STATE UNIVERSITY \\ OREGON STATE UNIVERSITY \\ STANFORD UNIVERSITY \\ UNIVERSITY OF ARIZONA \\ UNIVERSITY OF BRITISH COLUMBIA \\ UNIVERSITY OF CALIFORNIA \\ UNIVERSITY OF HAWAII
}

\author{
UNIVERSITY OF MONTANA \\ UNIVERSITY OF NEVADA, RENO \\ UNIVERSITY OF OREGON \\ UNIVERSITY OF SOUTHERN CALIFORNIA \\ UNIVERSITY OF UTAH \\ UNIVERSITY OF WASHINGTON \\ WASHINGTON STATE UNIVERSITY
}

The supporting Institutions listed above contribute to the cost of publication of this Journal, but they are not owners or publishers and have no responsibility for its contents or policies.

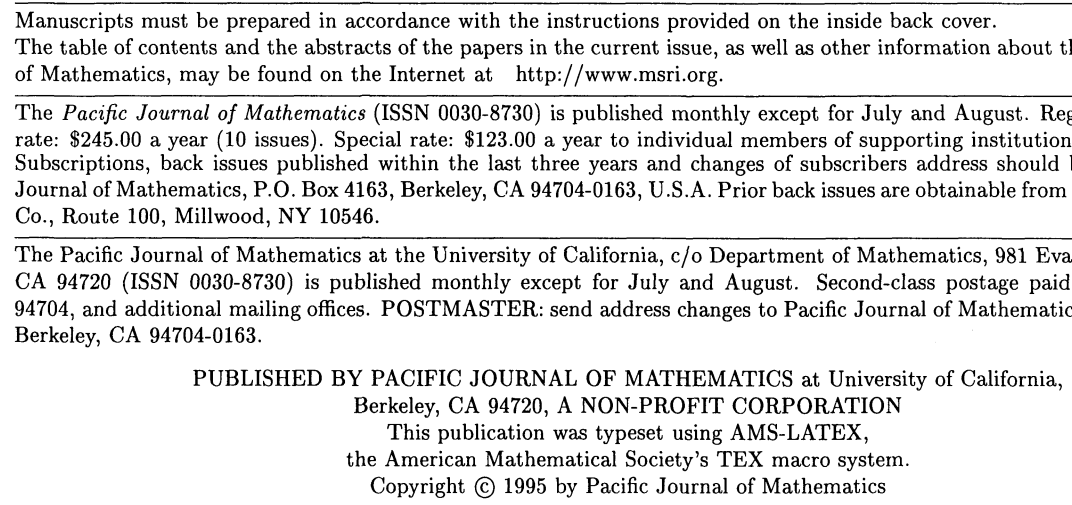




\section{PACIFIC JOURNAL OF MATHEMATICS}

\section{Volume $173 \quad$ No. $1 \quad$ March 1996}

Isometric immersions of $H_{1}^{n}$ into $H_{1}^{n+1}$

KINETSU ABE

Rotationally symmetric hypersurfaces with prescribed mean curvature

MARIE-FRANÇOISE BIDAUT-VÉRON

The covers of a Noetherian module

JIAN-JUN CHUAI

On the odd primary cohomology of higher projective planes

MARK FoSKEY and MiCHAEL DAVID SLACK

Unit indices of some imaginary composite quadratic fields. II

MiKinito HiRABAYASHI

Mixed automorphic vector bundles on Shimura varieties

MIN Ho LeE

Trace ideal criteria for Toeplitz and Hankel operators on the weighted Bergman

spaces with exponential type weights

PENG LIN and RICHARD ROCHBERG

On quadratic reciprocity over function fields

KATHY DONOVAN MERRILL and LYNNE WALLING

$\left(A_{2}\right)$-conditions and Carleson inequalities in Bergman spaces

TAKAHIKO NAKAZI and MASAHIRO YAMADA

A note on a paper of E. Boasso and A. Larotonda: "A spectral theory for solvable Lie 173 algebras of operators"
C. Отт

Tensor products with anisotropic principal series representations of free groups

Carlo Pensavalle and Tim Steger

On Ricci deformation of a Riemannian metric on manifold with boundary

YING SHEN

The Weyl quantization of Poisson $S U(2)$

Albert Jeu-Liang Sheu

Weyl's law for $S L(3, \mathbb{Z}) \backslash S L(3, \mathbb{R}) / S O(3, \mathbb{R})$

ERIC GeORge Stade and Dorothy IRENe Wallace (ANDREOli)

Minimal hyperspheres in two-point homogeneous spaces

PER TOMTER

Subalgebras of little Lipschitz algebras

NiKOLAI ISAAC WEAVER 